\title{
Extrapolating significance of text-based autonomous vehicle scenarios to multimedia scenarios and implications for user-centered design
}

\author{
Kwame Porter Robinson ${ }^{1}$, Lionel Robert ${ }^{1}$, Ron Eglash ${ }^{1}$
}

\begin{abstract}
Extrapolation from low-fidelity design iterations is especially critical in HRI. An initial proposal for low-fidelity to higher fidelity extrapolation is developed using insights from cognitive multimedia learning theory to account for the effects of prototype medium and three types of cognitive demands. Inspired by Donald Norman and others, our proposal leverages tightly controlled and multi-authored scenarios through crowdsourcing to create additional potential evidence as a kind of experimental "stress test." We motivate our proposal by investigating the intersection of emotion and human control, which is understudied outside of autonomous vehicles (AV) and HRI research. Evidence for positively moderated emotional effects in text-based AV scenarios as well as tentative evidence for our extrapolation proposal are identified.
\end{abstract}

\section{INTRODUCTION}

The importance of extrapolation from design iterations can not be overstated. In industry, user experience design matched the pace of Moore's Law because of faster design iterations caused by extrapolation from low fidelity prototypes [1]. Human-robot interaction (HRI) research has long used human-centered design (HCD) [2], [3], [4] since interaction success is largely determined by human perception. The importance and effort saved by reliable extrapolation at lower fidelity to high fidelity prototypes is even greater in HRI and autonomous vehicles (AV) research. Donald Norman, famous designer, prominent advocate (and critic) of $\mathrm{HCD}$, noticed that HCD created design solutions that were not radical and that radical designs were often created without a design process [5]. Following a field review of video game development and radical design change in technology, Norman and Verganti proposed "meaning change" as a philosophy for improving design research: "[o]ne promising direction in the development of radical innovation is to modify the HCD process to require simultaneous develop of multiple ideas and prototypes." [5, p. 96] Grappling with multiple perspectives allows the HCD designer to iterate towards likely global maxima in the design space because they are forced to deeply extrapolate from the eyes of others. Extrapolation from design iterations to global design maxima remains just as important in HRI research. As illustrated in Figure 1, our first contribution is an initial proposal for design-driven extrapolation of statistical significance from low-fidelity to high-fidelity HRI related prototypes. Although the effects of prototype medium within HRI have been investigated, associated theory has been minimal. We draw from an educational instruction theory to explain when extrapolation from low fidelity prototypes is and is not likely. Key to

\footnotetext{
${ }^{1}$ School of Information at University of Michigan, Ann Arbor, MI, USA $\{$ kwamepr, lprobert, eglash\}@umich.edu
}

our extrapolation proposal are multi-authored scenarios as a method for surfacing additional meaning and stress testing initial prototype findings at the same level of fidelity. This matches broader trends of greater extrapolation from reduced fidelity prototypes. The method may also support HRI researchers seeking unanticipated but superior designs.

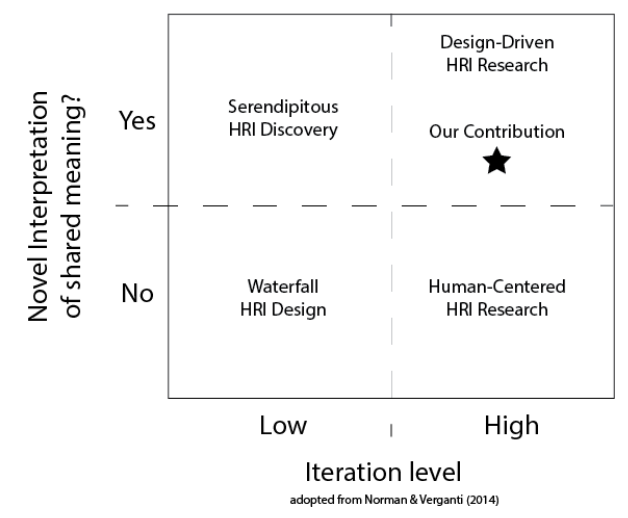

Fig. 1. Our extrapolation proposal in HRI design context

To motivate our investigation beyond $\mathrm{HCD}$ and into design-driven research we consider emotional interaction for sites of critical HRI control: autonomous vehicles. Arguments from legal culpability in finance, erroneous algorithmic decision making and preference modeling algorithms support the importance of human guided control of machines [6], [7], [8]. However, the autonomous vehicles literature represents one of the few areas where emotion and human guided control intersect. Reflecting upon experiences, or cognitive elaboration, is a daily phenomena but its effects in HRI scenarios is largely under investigated [9]. Our second contribution is to investigate elusive emotional environmental phenomena in HRI contexts, where we find evidence for positive moderated emotional effects.

\section{Related Work And Theoretical Development}

Our understanding of the proposed moderated relationship of cognitive elaboration, multi-authorship, emotion was motivated by cognitive science theory. In our main contribution, using concepts from educational instruction theory, the cognitive theory of multimedia media learning (multimedia learning theory) [10], we propose an initial extrapolation method from low to higher fidelity prototypes. Recognizing that HRI inherently contains vivid perceptual elements, we first review theory on vividness and emotion. Then we examine existing literature on low-fidelity extrapolation 
and identify how multimedia learning theory can address existing gaps. Important to both cognitive elaboration and our extrapolation proposal, we detail a method of controlled multi-authored scenario construction. Motivated by cognitive theory, we propose and justify a hypothesis that multiauthorship will moderate positive change in emotion. Finally we present an initial theoretical proposal that justifies extrapolating text-based scenario significance to other multimedia.

\section{A. Vividness and Emotion}

We study emotional induction and because vividness impacts emotion it is important to consider. Vividness, or attentional salience [11], is a conscious and salient quality of subjective experience that is often created when novel situations are encountered. A definition common in social psychology literature are those experiences “... likely to attract and hold our attention and to excite the imagination to the extent that it is emotionally interesting, concrete and imagery-provoking, and proximate in a sensory, temporal, or spatial way" [12]. Because HRI experiences are historically novel they necessarily contain vivid attributes [9]. For experimental subjects, novel HRI experiences include experiments involving prototypes although this may change as robot interaction becomes more common [13], [14]. The experimental psychology literature has extensively studied and constructed vivid stimuli [15] to create emotion manipulation conditions, including text-based scenarios. Vivid perceptual details associated with positive and negative emotion are remembered with greater clarity in psychophysical studies [16]. In our text-based AV experiments vividness self-report ratings after the experiment provide evidence that vivid experiences took place. Because vividness can be difficult to control [17] in this work we assume random prevalence of vividness intensity and include it within a theoretical model drawn from the literature.

\section{B. Extrapolation and Cognitive Load in Multimedia Learn- ing Theory}

The literature on extrapolation from low-fidelity design iterations has not resulted in or drawn upon unified theory of medium effects [18], [19], [20], [21], [22] even though the effects of medium in HRI experiment have been studied [23]. Zhou and Rau use correlational evidence drawn from mixed fidelity experiments to argue for matching prototype fidelity to one or more stages of usability testing [18] but do not generalize their findings beyond video game controllers or account for biases identified in other work. Other work identifies cognitive biases caused by low-fidelity prototype comparison to actual systems [20], [22], including idealization effects and hindsight bias. Sauer and Sonderegger found that mobile phone implementation fidelity did not impact usability evaluation or task completion time, suggesting that "prototypes of reduced fidelity may be suitable for modeling the reference system [e.g full physical system]" [19]. However, although the effects of and, in some cases, extrapolation from low-fidelity prototypes have been studied, in HRI the theory is scant.
Even as experimental HRI subjects, humans continuously learn from experiences. If we view HRI experimentation as a form of carefully controlled design wherein subject learning does and does not occur then we can consider cognitive theory of multimedia learning [10], [24] in the educational instruction literature. Here the effect of medium, or multimedia, on learning is defined by a cognitive relationship, or cognitive load, between the five senses, limited short-term memory and the utility of perceived information. Just as importantly, mental processing driven by imagination connects cognitive load to sensory capacity because imagination, later referred to as cognitive elaboration, engages the sensory areas of the brain [25]. Three types of cognitive load exist: (a) mental processing related to learning, or germane load, (b) mental processing unrelated to learning, or extraneous load, and, mental processing demanded by the media itself, or intrinsic load. The theory argues that limited short-term memory and sensory processing create a tradeoff between germane and extraneous load while the medium itself incurs a fixed intrinsic demand. Described as an additive relationship with finite bounds, when any combination of loads exceeds a subject's working memory then their experience of learning stops [10]. We describe in greater detail elsewhere how the tradeoff can be modelled and utilized to extrapolate the effects of a prototype using evidence from varied cognitive load experiments. Because experimental HRI settings are not typically purely educational they are likely to contain a mix of germane and extraneous loads along the intrinsic load of the prototype.

\section{Using Multi-Authorship to increase Cognitive Elabora- tion and relationship to Cognitive Load}

The mental process of reflecting upon, extrapolating from and filtering conveyed experience is cognitive elaboration [26]. Cognitive elaboration is important in HRI because humans cognitively reflect upon and filter vivid HRI experiences that are largely initially understood unconsciously. Yet it is unknown how cognitive elaboration affects other areas of subjective HRI experience, including emotion.

Under a multimedia learning theory perspective, cognitive elaboration is caused by processing germane and extraneous cognitive loads that inspire mental reflection. For the case of low-fidelity text-based prototypes, writers holding different viewpoints than the reader will generally reflect unexpected semantics and pragmatics in their writing. Content authored by multiple authors, or multi-authorship, contain a greater variety of unexpected information that increases the level of cognitive elaboration. For autonomous vehicles, vehicle driving and driving styles are controversial partially because they surface intense attitudes uniquely based on subjective experience and viewpoints [27], [28]. We expect that imagining autonomous driving scenarios from another perspective but written in first person will require greater cognitive elaboration because of strongly held driving attitudes. There is evidence of external and ecological validity of emotional text-based vignettes, or emotional short stories, to real life physical scenarios in the psychology appraisal literature [29]. 
By instructing participants to imagine themselves within first person vignettes of multiple or single authorship that they read out loud we investigate cognitive elaboration within emotional autonomous vehicle scenarios. When carefully controlled, we expect multi-authorship to be a method of increasing germane and extraneous reflective load, or cognitive elaboration, while keeping intrinsic load fixed. Figure 2 illustrates a constrained user interface for collecting crowdsourced AV scenario revisions that requires driving events, overall themes and several key words to be present.

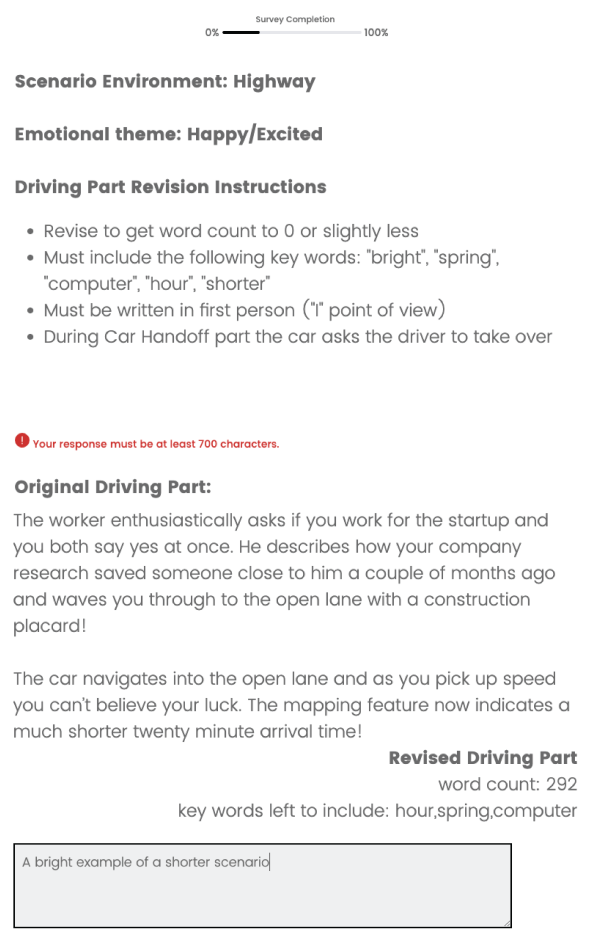

Fig. 2. Crowdsourcing interface for collecting controlled scenario revisions

D. Relationship between Cognitive Elaboration, Vividness and Emotion in Text-based AV scenarios

A small to medium moderation effect of vividness in emotional induction for positive emotion and not for negative emotion was found in a meta-analysis of marketing persuasion literature [30]. This difference in valence can be explained by the cognitive elaboration hypothesis [31] together with negatively valenced information hampering cognitive processing [32]. When cognitive elaboration is high the impact of vividness is high and when cognitive elaboration is low the impact of vividness is non-significant. The multi-authorship experiment will show that the emotional AV scenario induction is moderated by cognitive elaboration. We expect the multi-authorship condition to create a state of high cognitive elaboration that moderates a positive change in valence for positive scenarios. A greater number of positive emotion inductions would be evidence of the moderation effect, illustrated in Figure 3.

Hypothesis 1: That multi-authored vignettes will cause more positive emotion induction than single authored vignettes

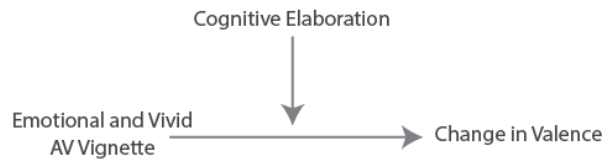

Fig. 3. Cognitive elaboration moderates the change in valence induced by emotional vividness

\section{E. Using Cognitive Elaboration to Extrapolate towards High-fidelity Significance}

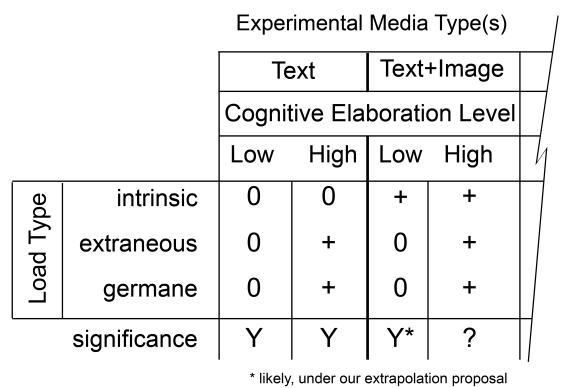

Fig. 4. Extrapolation from high and low prototypes using cognitive elaboration

Under multimedia learning theory, an HRI experiment contains germane, extraneous and intrinsic load (or cognitive demands) in relation to the dependent variable of interest. In repeated experiments involving the same medium the intrinsic load is held constant. High levels of cognitive elaboration increase extraneous and germane load through multi-authorship to create another, potentially more stringent, test of the dependent variable of interest. This design-driven test for HRI is founded upon the importance of multiple meaning in design-driven research per Norman and Verganti [5].

Our proposal is unique in that it incorporates multiauthorship to carefully redesign prototypes (text-based AV scenarios in this work) to produce additional design evidence at the same level of fidelity as a form of "stress test." Within the same experimental medium, if an outcome of interest remains significant at low and high cognitive levels then multimedia learning theory suggests that it will also be significant in a medium of higher intrinsic load at a low cognitive level elaboration level, as illustrated in Figure 4. This is because the additive cognitive load between the two tests remains similar, allowing a theoretical relationship to persist. However, if an outcome of interest is significant at only one of the cognitive levels in a low-fidelity experiment then we would expect mixed results at a higher fidelity. Finally, if the outcome of interest is not significant at all then we would expect that medium related biases were unaccounted for or that high-fidelity significance will not be found. Following [5], this kind of evidence can lead to revolutionary understanding and easier identification of "global maxima" in the space of HRI. 


\section{EXPERIMENTAL STUDY}

\section{A. Sample}

Participants were recruited through a non-probability national U.S. convenience sample from the Prolific crowdsourcing platform. For the single authored experiment, a total of 45 participants (18 female, 2 prefer not to say) with an average age 25 - 34 years old. For the multi-authored experiment, a total of 43 participants (18 female) with an average age of 25 - 34 years old. Participants were assigned to only one experiment. Participants were paid $\$ 7.25$ an hour for their participation in the $45 \mathrm{~min}$ to $90 \mathrm{~min}$ experiment. This research was approved by the institutional review board at the University of Michigan. We obtained informed consent from each participant.

\section{B. Method}

To test the hypotheses we conducted a controlled experiment where participants participated in trials where they (a) provided a pretest rating of their emotion state along valence and arousal dimensions [33], (b) verbally read aloud an emotional valenced text based autonomous vehicle scenario and (c) provided a posttest rating of emotion. After all experimental trials the participant provided a summary rating of scenario vividness along with and other qualitative feedback. The experimental condition of authorship was manipulated through the number of writers.

\section{Design}

This study included two studies. One study included single-authored vignettes only and the other study included multi-authored vignettes only. Participants were exposed to two trials that included an unique negatively valenced or unique positively valenced vignette. Randomized blocks examined the impact of authorship within the experiment. The task was a pretest-posttest emotional induction task where the participant first reported emotional valence and arousal using a self assessment manikin [34], read aloud a positive or negative valenced text based autonomous vehicle scenario (a vignette) and then again reported emotional valence and arousal. The within-subjects component controlled for differences in subject emotional response by accounting for individual variability. The experimental design is illustrated in Figure 5.

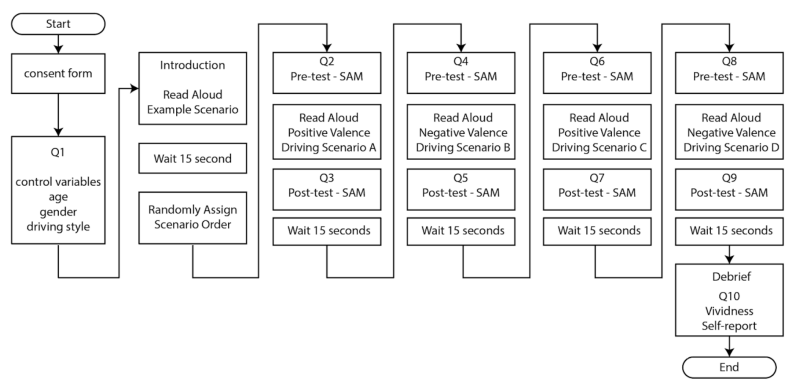

Fig. 5. Experimental design

\section{Measures}

We measure emotional valence and arousal with Self Assessment Manikin (SAM) [34] and this measure has been used in prior autonomous vehicle studies [35].

\section{E. Variables}

Authorship was manipulated for vignette creation to create two levels, single and multiple authorship, for positive and negative emotional scenarios as independent variables. The dependent pretest-posttest emotion valence as the study dependent variable.

Uniquely, we create multi-authorship by allowing authors to revise single authored vignettes in a constrained crowdsourced creative revision task and we use their creative work to synthesize a new vignette. In the crowdsourced revision task, word omissions and inclusions support higher cognitive elaboration when synthesized with another author's word omissions and inclusions by juxtaposing different viewpoints. Each of the 4 original scenarios, positive and negative valenced scenarios, were rewritten by two workers for a total of 8 new scenarios. Participants were paid $\$ 7.25$ an hour for $20 \mathrm{~min}$ to $90 \mathrm{~min}$ of work.

\section{F. Task}
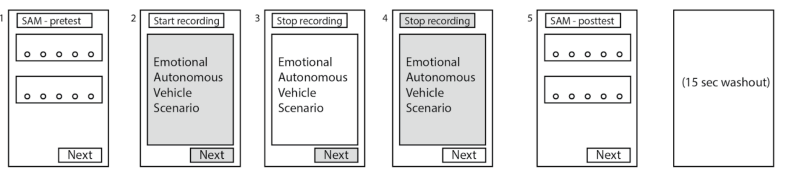

Fig. 6. Experimental task sequence

Referring to Figure 6, the experimental task was to (1) complete a pretest SAM, (2, 3 and 4) read out loud from beginning to end the provided vignette, (5) complete a posttest SAM and then wait 15 seconds for the next vignette. In step (2) the user presses start recording prior to reading the vignette. In step (3) the user reads the complete vignette and then presses "stop recording." In step (4) the user presses next to advance to the posttest SAM which is then completed. The 15 second wait acted as a washout period to allow user emotion to settle to a presumably more neutral level. Parts of the actual implementation are shown in Figure 7 that represent $(1,5)$ and the main screen where steps $(2,3$ and 4$)$ are carried out. A typical task took 10 minutes to complete.
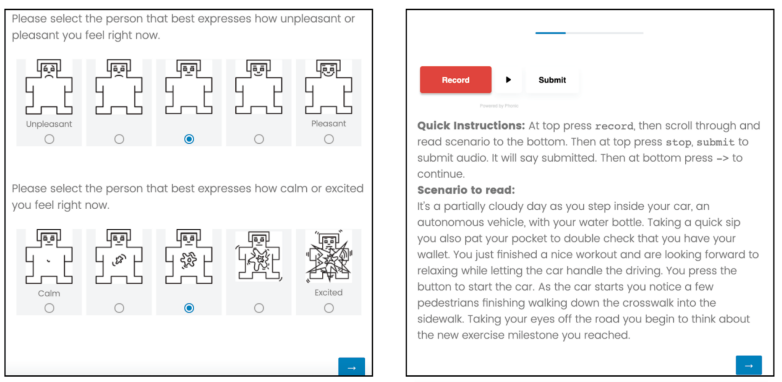

Fig. 7. Actual main task screens 


\section{RESULTS}

\section{A. Emotional Manipulation in High and Low Cognitive Elaboration}

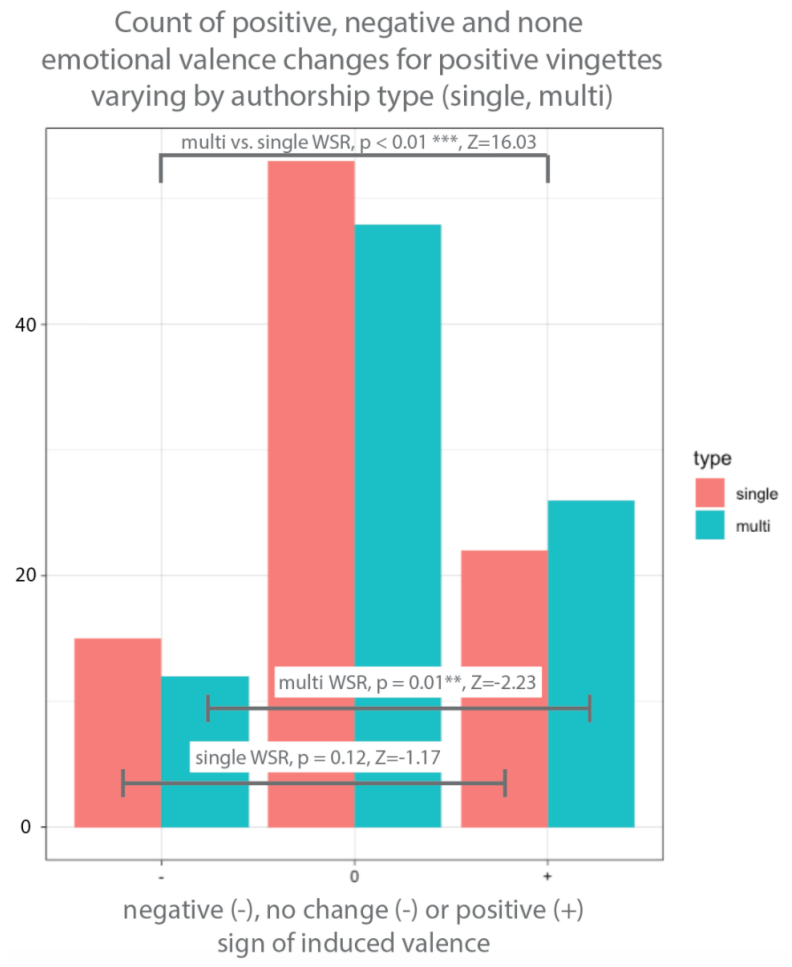

Fig. 8. Counts of change in valence for multi and single authorship

Experimental evidence that multi-authorship induced greater emotional manipulation was found. The barchart shown in Figure 8 with single authorship in pink and multi-authorship in blue shows a test of distributional shift using the Wilcoxon signed rank test over three emotional induction outcomes: negative, no change and positive. A comparison of multi-authored and single authored differences for positive vignettes was significantly different (WSR $p$ $<0.01, Z=16.03$, Bonferroni corrected). Single authored vignettes failed to induce significant positive emotional changes. Hypothesis 1 was supported by significant evidence for multi-authorship and insignificant evidence for single authored vignettes. Negative vignettes in both single and multi authored experiments showed significant negative valence induction (both $p<0.01$ ). To account for vividness distributions as being a confounder we conducted a posthoc test on self-reported vividness. Both multi-authored and single authored experiments had the same distribution of selfreported vividness per two sample Kruskal Wallis test $\left(\chi^{2}\right.$ $=0.60, \mathrm{df}=1, \mathrm{p}=0.44)$. Multi-authorship causes higher levels of cognitive elaboration and interacts with vividness in the positive condition as theorized. In summary, we found support for our hypothesis that cognitive elaboration causes significant emotional manipulation in text-based AV scenarios.

\section{DISCUSSION}

That multi-authored stimuli and not single authored stimuli showed significant effects in positive AV text-based scenarios directly promotes the importance of viewpoints in low fidelity HRI study. The importance of perspective is well known in the science, technology and society (STS) literature, including the concept of unexpected information from counter-experts [36], as well as promoted by Donald Norman [5] for design-driven research.

A post-hoc review of positive and negative emotion induction in higher fidelity automated (2) and manual (1) driving experiments indicate greater robustness for negative emotion inductions: greater number of inductions [37], range of induction [38] and inter-rater reliability [39]. A broad literature review indicates that scenario based methods for emotional induction are more robust in negative than positive cases [40]. Although further experiments are needed, for our proposed extrapolation method this partial evidence reflects the kind of mixed results one might expect when an effect is found at one but not both levels of cognitive elaboration.

As future work, single authored vignettes revised by the same author could be compared to multi-authored vignettes to better examine the hypothesized effects of multiple viewpoints for cognitive elaboration. Following our multimedia extrapolation proposal, extrapolation from text to image+text or even video+text format should be tested.

\section{CONCLUSIONS}

In this study we investigated emotional responses to text based autonomous vehicle scenarios and proposed a lowfidelity design-driven extrapolation method. Our findings contribute to the affective HRI literature in several ways. First, we showed that a vividness-cognitive elaboration relationship existed for positive AV scenarios. This finding is an important contribution because it provides support for elusive emotional environmental phenomena in HRI contexts where users engage in higher cognitive elaboration. Our second contribution is a proposal for extrapolating lowfidelity findings from text-based scenarios to higher fidelity multimedia scenarios. Although tentative, this proposal may allow the HRI experimenter to use design-driven research to better identify "global maxima" in HRI design through multiple perspectives providing additional stress tests as evidence. Additional research is needed to isolate the true effect of multi-authored stimuli as well as experimentally test our extrapolation proposal.

\section{REFERENCES}

[1] I. Armstrong, "The Evolution of UX Process Methodology," Mar. 2018.

[2] J. Scholtz, "Theory and evaluation of human robot interactions," in 36th Annual Hawaii International Conference on System Sciences, 2003. Proceedings of the, Jan. 2003, pp. 10 pp.-.

[3] B. Pokornia, J. Zwerinaa, and M. Hämmerle, "Human-centered design approach for manufacturing assistance systems based on Design Sprints," Procedia CIRP, vol. 91, pp. 312-318, Jan. 2020, publisher: Elsevier.

[4] G. A. Boy, The Handbook of Human-Machine Interaction: A HumanCentered Design Approach. CRC Press, Nov. 2017, google-Books-ID t2kQEAAAQBAJ. 
[5] D. A. Norman and R. Verganti, "Incremental and Radical Innovation: Design Research vs. Technology and Meaning Change," Design Issues, vol. 30, no. 1, pp. 78-96, Jan. 2014.

[6] R. P. Buckley, D. A. Zetzsche, D. W. Arner, and B. W. Tang, "Regulating artificial intelligence in finance: Putting the human in the loop," Sydney Law Review, The, Mar. 2021.

[7] T. E. Allen, M. Chen, J. Goldsmith, N. Mattei, A. Popova, M. Regenwetter, F. Rossi, and C. Zwilling, "Beyond Theory and Data in Preference Modeling: Bringing Humans into the Loop," in Algorithmic Decision Theory, ser. Lecture Notes in Computer Science, T. Walsh, Ed. Cham: Springer International Publishing, 2015, pp. 3-18.

[8] M. De-Arteaga, R. Fogliato, and A. Chouldechova, "A Case for Humans-in-the-Loop: Decisions in the Presence of Erroneous Algorithmic Scores," in Proceedings of the 2020 CHI Conference on Human Factors in Computing Systems, ser. CHI '20. New York, NY, USA: Association for Computing Machinery, Apr. 2020, pp. 1-12.

[9] J. E. Michaelis, M. C. Gombolay, M. M. Graaf, and T. Kanda, "Novelty Effects in Human-Robot Interaction," Dec. 2020

[10] R. E. Mayer, "Cognitive theory of multimedia learning." in The Cambridge handbook of multimedia learning, $2 n d$ ed., ser. Cambridge handbooks in psychology. New York, NY, US: Cambridge University Press, 2014, pp. 43-71.

[11] T. Parr and K. J. Friston, "Attention or salience?" Current Opinion in Psychology, vol. 29, pp. 1-5, Oct. 2019.

[12] R. Nisbett and L. Ross, Human inference: Strategies and shortcomings of social judgment. Institute for Social Research (ISR), 1985.

[13] H.-I. Liao, S.-L. Yeh, and S. Shimojo, "Novelty vs. Familiarity Principles in Preference Decisions: Task-Context of Past Experience Matters," Frontiers in Psychology, vol. 2, 2011, publisher: Frontiers.

[14] C. V. Smedegaard, "Reframing the Role of Novelty within Social HRI: from Noise to Information," in 2019 14th ACM/IEEE International Conference on Human-Robot Interaction (HRI), Mar. 2019, pp. 411420, iSSN: 2167-2148.

[15] K. Riddle, "Vividness and Salience," in The International Encyclopedia of Media Psychology. John Wiley \& Sons, 2020, pp. 1-9, _eprint: https://onlinelibrary.wiley.com/doi/pdf/10.1002/9781119011071.iemp0063.

[16] R. M. Todd, D. Talmi, T. W. Schmitz, J. Susskind, and A. K. Anderson, "Psychophysical and Neural Evidence for Emotion-Enhanced Perceptual Vividness," The Journal of Neuroscience, vol. 32, no. 33, pp. 11201-11212, Aug. 2012.

[17] R. L. Collins, S. E. Taylor, J. V. Wood, and S. C. Thompson, "The vividness effect: Elusive or illusory?" Journal of Experimental Social Psychology, vol. 24, no. 1, pp. 1-18, Jan. 1988.

[18] X. Zhou and P.-L. P. Rau, "Determining fidelity of mixed prototypes: Effect of media and physical interaction," Applied Ergonomics, vol. 80, pp. 111-118, Oct. 2019

[19] J. Sauer and A. Sonderegger, "The influence of prototype fidelity and aesthetics of design in usability tests: Effects on user behaviour, subjective evaluation and emotion," Applied Ergonomics, vol. 40, no. 4, pp. 670-677, July 2009.

[20] L. Christoforakos and S. Diefenbach, "Idealization Effects in UX Evaluation at Early Concept Stages: Challenges of Low-Fidelity Prototyping," in Advances in Usability, User Experience and Assistive Technology, ser. Advances in Intelligent Systems and Computing, T. Z. Ahram and C. Falcão, Eds. Cham: Springer International Publishing, 2019, pp. 3-14.

[21] Y. Earnshaw, A. A. Tawfik, and M. Schmitz, "User Experience Design," in Foundations of Learning and Instructional Design Technology, Jan. 2017.

[22] S. Diefenbach, W.-C. Chien, E. Lenz, and M. Hassenzahl, "Prototypen auf dem Prüfstand. Bedeutsamkeit der Repräsentationsform im Rahmen der Konzeptevaluation," vol. 12, no. 1, pp. 53-63, Apr. 2013, publisher: Oldenbourg Wissenschaftsverlag GmbH Section: i-com.

[23] Q. Xu, J. Ng, O. Tan, Z. Huang, B. Tay, and T. Park, "Methodological Issues in Scenario-Based Evaluation of Human-Robot Interaction," International Journal of Social Robotics, vol. 7, no. 2, pp. 279-291, Apr. 2015 .

[24] R. Brunken, J. L. Plass, and D. Leutner, "Direct Measurement of Cognitive Load in Multimedia Learning," Educational Psychologist, vol. 38, no. 1, pp. 53-61, Mar. 2003.

[25] J. Pearson, "The human imagination: the cognitive neuroscience of visual mental imagery," Nature Reviews Neuroscience, vol. 20, no. 10, pp. 624-634, Oct. 2019, number: 10 Publisher: Nature Publishing Group.

[26] J. Anderson and G. Bower, Human Associative Memory, 1st ed., 1980.
[27] G. Miller and O. Taubman-Ben-Ari, "Driving styles among young novice drivers-the contribution of parental driving styles and personal characteristics," Accident; Analysis and Prevention, vol. 42, no. 2, pp. 558-570, Mar. 2010.

[28] K. A. Suhr and S. M. Nesbit, "Dwelling on 'Road Rage': The effects of trait rumination on aggressive driving," Transportation Research Part F: Traffic Psychology and Behaviour, vol. 21, pp. 207-218, Nov. 2013.

[29] M. D. Robinson and G. L. Clore, "Simulation, Scenarios, and Emotional Appraisal: Testing the Convergence of Real and Imagined Reactions to Emotional Stimuli," Personality and Social Psychology Bulletin, vol. 27, no. 11, pp. 1520-1532, Nov. 2001, publisher: SAGE Publications Inc.

[30] J. Blondé and F. Girandola, "Revealing the elusive effects of vividness: a meta-analysis of empirical evidences assessing the effect of vividness on persuasion," Social Influence, vol. 11, no. 2, pp. 111-129, Apr. 2016, publisher: Routledge _eprint: https://doi.org/10.1080/15534510.2016.1157096

[31] A. L. McGill and P. Anand, "The Effect of Vivid Attributes on the Evaluation of Alternatives: The Role of Differential Attention and Cognitive Elaboration," Journal of Consumer Research, vol. 16, no. 2, pp. 188-196, 1989, publisher: Oxford University Press.

[32] O. Megalakaki, U. Ballenghein, and T. Baccino, "Effects of Valence and Emotional Intensity on the Comprehension and Memorization of Texts," Frontiers in Psychology, vol. 10, 2019, publisher: Frontiers.

[33] J. A. Russell, "A circumplex model of affect," Journal of Personality and Social Psychology, vol. 39, no. 6, pp. 1161-1178, 1980, place: US Publisher: American Psychological Association.

[34] Bradley and Lang, "Measuring Emotion: The Self-Assessment Manikin and the Semantic Differential," Mar. 1994, iSSN: 0005-7916 Issue: 1 Library Catalog: pubmed.ncbi.nlm.nih.gov Publisher: J Behav Ther Exp Psychiatry Volume: 25.

[35] N. Du, F. Zhou, E. Pulver, D. M. Tilbury, L. P. Robert, A. K Pradhan, and X. J. Yang, "Examining the Effects of Emotional Valence and Arousal on Takeover Performance in Conditionally Automated Driving," arXiv:2001.04509 [cs], Jan. 2020, arXiv: 2001.04509.

[36] L. D. A. Williams and S. Moore, "Guest Editorial: Conceptualizing Justice and Counter-Expertise," Science as Culture, vol. 28, no. 3, pp. 251-276, July 2019, publisher: Routledge _eprint: https://doi.org/10.1080/09505431.2019.1632820.

[37] N. Du, F. Zhou, E. M. Pulver, D. M. Tilbury, L. P. Robert, A. K. Pradhan, and X. J. Yang, "Examining the effects of emotional valence and arousal on takeover performance in conditionally automated driving," Transportation Research Part C: Emerging Technologies, vol. 112, pp. 78-87, Mar. 2020.

[38] M. Jeon, B. N. Walker, and J.-B. Yim, "Effects of specific emotions on subjective judgment, driving performance, and perceived workload," Transportation Research Part F: Traffic Psychology and Behaviour, vol. 24, pp. 197-209, May 2014

[39] N. Alyuz, S. Aslan, J. Healey, I. J. Alvarez, and A. A. Esme, "Towards Understanding Emotional Reactions of Driver-Passenger Dyads in Automated Driving," in 2018 13th IEEE International Conference on Automatic Face Gesture Recognition (FG 2018), May 2018, pp. 585592.

[40] E. Siedlecka and T. F. Denson, "Experimental Methods for Inducing Basic Emotions: A Qualitative Review," Emotion Review, vol. 11, no. 1, pp. 87-97, Jan. 2019, publisher: SAGE Publications. 